\title{
Las relaciones de equivalencia como criterio de análisis de la pertinencia científica de categorías analíticas
}

\author{
Tiberio Pérez Manrique* y Ernesto L. Ravelo C. \\ Universidad Católica de Colombia
}

Recibido, mayo 1/2017

Concepto de evaluación, mayo 22/2017

Aceptado, mayo 23/2017
Referencia: Pérez Manrique, T. \& Ravelo Contreras, E.L. (2017). Las relaciones de equivalencia como criterio de análisis de la pertinencia científica de categorías analíticas. Acta Colombiana de Psicología. 20(2), 253-261. doi: http:// www.dx.doi.org/10.14718/ACP.2017.20.2.12

\section{Resumen}

El objetivo del presente trabajo es proponer las relaciones de equivalencia como criterio estructural o de ordenamiento analítico mediante el cual se puedan delimitar las funciones del conocimiento requeridas en una actividad científica. Se parte de considerar al conocimiento como un fenómeno estudiado por la psicología y cuya explicación requiere de dar cuenta de la manera en que se establecen, organizan y actualizan las funciones conductuales o del conocimiento. De este modo, se considera que las tareas de igualación a la muestra permiten comprender el modo de abstraer las categorías analíticas que estructuran científicamente el conocimiento de la realidad. Cuando se usan estos procedimiento, se derivan nuevas relaciones sin la necesidad de un entrenamiento directo y que no pueden explicarse con base en principios de generalización de estímulos, sino, más bien, como relaciones equivalentes diferenciadas mediante los entrenamientos previos y que adquieren un cierre en una categoría analítica cuando se abstraen los criterios categoriales o, si se quiere, cuando estas relaciones se nominan. Así, al categorizarse o nominarse, estas trascienden la situación — porque nominar se entiende como un ajuste a criterios convencionales - y una vez que las interacciones situacionales se nominan, regulan todas las prácticas sociales, incluidas las prácticas del conocimiento científico.

Palabras clave: Conocimiento científico, relaciones de equivalencia, categoría analítica.

\section{Equivalence relations as an analysis criterion of scientific relevance of analytical categories}

\begin{abstract}
The aim of this paper is to propose equivalence relations as a structural criterion or analytical order, through which the functions of knowledge required in a scientific activity are delimited. It begins by considering knowledge as a phenomenon studied by psychology, whose explanation requires giving an account of the way in which such behavioral or knowledge functions are established, organized and updated. It is considered that the procedures of matching-to-sample allow understanding the way of abstracting the analytical categories that scientifically structure the knowledge of reality. When using these procedures, new relationships are derived that without the need for direct training, cannot be explained based on principles of stimulus generalization, but rather as equivalent relations differentiated through previous training. They get a closure in an analytical category, once the categorical criteria are abstracted or if desired, when these relationships are nominated. And when categorized or nominated, they transcend the situation, since nominating is understood as an adjustment to conventional criteria. Once situational interactions are nominated, they regulate all social practices, including practices of scientific knowledge.

Key words: Scientific knowledge, equivalence relations, analytical category.
\end{abstract}

* Facultad de Psicología: Avenida Caracas No. 46 - 40, Tel. 3277300 Ext. 5056.tperez@ucatolica.edu.co; elravelo@ucatolica.edu.co Este trabajo es el resultado de la línea de investigación con la formación científica, que los autores han venido presentando en la publicación de artículos. 


\title{
As relaçóes de equivalência como critério de análise da pertinência científica de categorias analíticas
}

\begin{abstract}
Resumo
O objetivo deste trabalho é propor as relações de equivalência como critério estrutural ou de ordenamento analítico pelo qual possam ser delimitadas as funções do conhecimento requeridas numa atividade científica. Parte-se de considerar o conhecimento como um fenômeno estudado pela psicologia e cuja explicação requer evidenciar a maneira na qual são estabelecidas, organizadas e atualizadas as funções comportamentais ou do conhecimento. Desse modo, considera-se que as tarefas de igualação à amostra permitem compreender o modo de abstrair as categorias analíticas que estruturam cientificamente o conhecimento da realidade. Quando são usados esses procedimentos, são derivadas novas relações que, sem a necessidade de um treinamento direto, não podem ser explicadas com base em princípios de generalização de estímulos, mas sim como relações equivalentes diferenciadas mediante os treinamentos prévios e que adquirem uma delimitação numa categoria analítica quando são abstraídos os critérios categoriais ou, se se quiser, quando essas relações são nominadas. Assim, ao serem categorizadas ou nominadas, elas transcendem a situação — porque nominar se entende como um ajuste a critérios convencionais- e, uma vez que as interações situacionais são nominadas, regulam todas as práticas sociais, incluídas as práticas do conhecimento científico.

Palavras-chave: categoria analítica, conhecimento científico, relações de equivalência.
\end{abstract}

\section{INTRODUCCIÓN}

La ciencia, como categoría analítica y práctica social, ha derivado su significado de los usos cotidianos del lenguaje. Padilla (2014) afirma que "[...] la ciencia ha sido objeto de estudio de la filosofía, la sociología, la historia, la economía, etc., y solamente a partir de los años 60, de la psicología" (p. 35). En el uso cotidiano, la palabra ciencia contiene múltiples significados, pues con esta se hace referencia al conocimiento especializado que se tiene respecto de un segmento de la realidad, además de que se usa para referirse a los procedimientos que se emplean para establecer y validar los distintos conocimientos.

En ocasiones se usa el concepto de ciencia como sinónimo de teoría o teorías científicas; $y$, de manera general, la palabra ciencia agrupa los productos avalados y categorizados por una comunidad como productos científicos; el conjunto de actividades que una comunidad avala como actividades científicas; y una justificación de las actividades consideradas como científicas, tales como los procedimientos y las teorías. Cada una de estas tiene como propósito hacer comprensibles los fenómenos de la naturaleza, de manera que con base en esta comprensión se establecen los distintos modos de relación u orientaciones respecto de las materias de las que tratan las teorías científicas.

Ahora bien, cuando se habla de productos científicos por lo general se hace referencia a las teorías, a las leyes científicas o a las aplicaciones derivadas de la teoría; y cuando se habla de actividades científicas se les trata como competencias o pensamiento científico, lo cierto es que aún no existen criterios con los cuales se puedan delimitar las actividades científicas de aquellas que no lo son.
Vale recordar que este no es un problema nuevo en la historia del conocimiento, pues la validez del conocimiento fue un problema para los griegos, quienes buscaron criterios para hacer distinción entre doxa y conocimiento verdadero (Patón, 1993); al igual que para Descartes, quien intentó fundamentar todo conocimiento en la entidad metafísica res cogitans, caracterizada porque en ella se realizaban la operaciones de la razón como fundamento de la verdad. Posteriormente, Kant (1781/2016), no satisfecho con la solución de Descartes, acomete la tarea de establecer los principio fundamentales de la razón y traslada la discusión metafísica sobre qué es la razón hacia la de cuáles son las condiciones necesarias para su expresión y en qué se fundamenta la propia razón. Así, Kant centra su análisis en las condiciones de validez de los juicios, pues es mediante estos que se expresa el conocimiento de la realidad. Mas tarde, los neokantianos interpretaron a Kant como un filósofo del lenguaje, y la razón se convirtió en las condiciones de lo expresable con sentido (Wittgestein, 1953).

Así, los análisis filosóficos han servido de base para la diversidad de aproximaciones científicas desde la psicología al problema del conocimiento (Piaget, 1977; Piaget \& García, 1982). Por otra parte, Skinner (1957/1981) se refirió a las actividades científicas como aquellas que una comunidad científica moldea y mantiene mediante contingencias de refuerzo y cuya naturaleza y principios no son distintos de los aplicados a la explicación de cualquier otra conducta.

Teniendo esto en cuenta, ¿cuál es entonces la distinción entre el conocimiento científico y otros tipos de conocimiento? Desde el pragmatismo (Pierce, 1935), es solo en las prácticas sociales que se establecen las diferencias, puesto que es en estas donde se delimitan las implicaciones de las 
distintas actividades, y no en unos principios a priori. Es decir que el conocimiento se inserta en estas prácticas como una forma de vida. Así mismo, dicho pragmatismo trajo a discusión la relación que puede darse entre la experiencia como forma "subjetiva" de conocimiento y las prácticas sociales efectivas reguladas lingüísticamente, además de que abrió la posibilidad de investigar científicamente la experiencia subjetiva 0 , mas bien, su modo de expresión.

Igualmente, el conocimiento científico como producto (teorías o leyes) no es distinto de otros productos de conocimiento, como los del conocimiento matemático, tecnológico, artístico o de otra clase. Como se sugirió antes, los griegos buscaron hacer una distinción entre los diferentes tipos de conocimiento, principalmente entre los conocimiento de la ciencia y de la creencia; en este sentido, como criterio de distinción propusieron diferentes tipos de justificación: las justificaciones formales - que tienen un criterio lógico-y las justificaciones desde la acción posible sobre la realidad - que, como se diría en la actualidad, corresponden a un criterio empírico-. Sin embargo, cualquiera que sea la justificación, esta no puede partir de ningún presupuesto de justificación dado que esto daría origen a una justificación dogmática o a una petición de principio.

Si bien los filósofos habían discutido ampliamente las posibilidades del conocimiento, fue a partir de Darwin $(1859 / 1983)$ y la teoría de la evolución por selección natural que comenzó el interés en el conocimiento como un proceso natural que sucede a los seres vivos y toma realidad en su acción - con especial referencia a los humanos-, y del que se podía dar cuenta mediante los métodos de la ciencia - el conocimiento como un hecho natural-. Teoría que contrasta con la visión teocéntrica de conocimiento Santo Tomás (1944-1945), entendida como la experiencia que se refleja en una mente trascendental que iluminaba y guiaba la acción - principalmente como guía de las acciones humanas interpretadas a la luz de supuestos morales y políticos como un deber a priori-, interpretación que además predeterminaba la finalidad del hombre más allá de este mundo.

Ahora, si bien la teoría de la evolución facilitó la naturalización del "pensamiento", el pensamiento como connatural al hombre terrenal requería hacer la siguiente precisión: debido a que los teóricos de la evolución hicieron un mayor énfasis en la evolución de las estructuras y prestaron poca atención a los elementos del contexto de vida del organismo como posibilitadores de la estructura - o como eventos disposicionales, para usar la conceptualización de Ryle (1949) - al hacer énfasis en la evolución de las estructuras se separó al organismo del ambiente y de sus modos de vida, en vez de verlo como una unidad interactiva.
Esta separación de los organismos de sus formas de vida ha influido profundamente en la forma de concebir al conocimiento como un fenómeno psicológico, lo que ha inducido, por ejemplo, a considerar que lo psicológico es una estructura que ayuda al organismo en su adaptación al ambiente; y así, al perderse de vista el contexto como condición necesaria para la ocurrencia de los fenómenos psicológicos, se opta por una explicación de estos fenómenos desde niveles teóricos distintos a los propiamente psicológicos, como sucede con la teoría de la evolución - tal como lo proponen las epistemologías evolucionistas - (Cambell, 1974; Lorenz, 1977; Pinker, 1997; Skinner, 1981).

Las epistemologías evolucionistas hicieron uso de los conceptos de filogenia y ontogenia a la hora de analizar el conocimiento y propusieron un paralelo entre el conocimiento como un producto, y el cerebro como el principal mecanismo de producción de conocimiento; en este enfoque se propone que el conocimiento es una función del cerebro - lo que se conoce como dualismo de propiedades - (Changeaux, 1985; Edelman, 1987).

Este tipo de propuestas indican que no se han comprendido los procesos seleccionistas que operan en la filogenia y su diferencia con los procesos de desarrollo ontogenético -que aunque dependen de la filogenia, no se encuentran necesariamente determinados por ella, pero ante los cuales también es viable aplicar la lógica seleccionista en un nivel distinto-. Así, La explicación seleccionista se ha aplicado de manera indiscriminada tanto a los procesos de diferenciación filogenética como ontogenética. Por ejemplo, cuando se afirma que el desarrollo ontogénico está ya codificado por los genes, sin tener en cuenta que la diferenciación estructural, más que venir ya prefigurada, depende de las condiciones presentes durante el desarrollo de un organismo que actualiza sus mecanismos de origen filogenético.

Tal vez es tiempo de abandonar la idea de las epistemologías tradicionales -el racionalismo y el empirismo, principalmente - que hacen una distinción entre el sujeto que conoce y el objeto de conocimiento como si fueran dos sustancias de naturaleza distinta, puesto que esta vía de análisis ha conducido a asumir supuestos tales como que el conocimiento es el producto de unos mecanismos biológicos - el cerebro-, lo que reduce todo proceso de conocimiento a la actividad que realiza un agente causal -el cerebro- o, peor aún, al considerar a las actividades de conocimiento como análogas de la actividad de un agente mental; lo que es simplemente apelar al dogmatismo insertado en un agente con capacidad de conocimiento — cabe aclarar que es distinto considerar la existencia de dos sustancias a considerar categorías distintas de análisis; una cosa es una categoría descriptiva y otra una categoría explicativa- . 
Si se abandona la idea de sujeto como entidad sustantiva se está ante la propuesta de Aristóteles, para quien lo psicológico - incluyendo el conocimiento- puede entenderse como actos, es decir que la interactividad es la característica de todo ser vivo, y eso que llamamos consciencia es la misma propiedad interactiva. Siendo actos, estos solo toman forma y se pueden conocer cuando se realizan o actualizan; y aun cuando las acciones psicológicas están en potencia - es decir, aunque no esté hablando en este momento, cuando hablo y hay un oyente que responde, es en este momento cuando el acto cobra interés psicológico y es distinto del "acto de sonar"-, las potencias se actualizan en relación a las condiciones de estimulación —no tendría mucho sentido hablar de un ojo que no ve, como no tendría sentido hablar de un sujeto que no actúa-.

A partir de esto, puede decirse que un análisis del conocimiento, más que centrarse en el análisis de un agente en cuya naturaleza esté el conocer - llámese mente o cerebro-, debe centrarse en los actos coordinados y reconocidos por una comunidad como actos de conocimiento.

\section{ACTOS DE CONOCIMIENTO}

Saber y conocer implican actos, pero no constituyen actos (Ribes, 2010). Por lo general, se usa el verbo saber para cuando se hace algo con algún fin, como cuando digo que sé montar bicicleta o que sé inglés. Por otro lado, el verbo conocer tiene un uso "declarativo", o se usa como un referente en un episodio de habla para especificarle algo a alguien, por ejemplo, cuando se dice "conozco quien arregla el teléfono". Sarmiento (2011) considera que

[...] la mayoría de aclaraciones sobre términos epistémicos se ha hecho en los últimos años en inglés con la dificultad de que en esta lengua no existe una diferencia entre los verbos creer y conocer, pues la palabra "know" abarca ambos. Cosa distinta ocurre en castellano (conocer y saber), alemán (kennen y wissen), francés (connaître y savoir) (p.81).

En el Latín, el verbo conocer tiene dos acepciones: gnoscere y scire, que significan "conocer por los sentido" y "conocer por la mente", respectivamente; significados que expresan las mismas concepciones de saber y conocer. En este orden, se puede decir que el conocer se refiere a la especificación de los haceres, mientras que el saber $-\mathrm{y}$ su especificación, el conocer- ocurre en un tiempo y un espacio distinto — desde el aquí y el ahora, y desde el allá y el entonces - como lo proponen Barnes y Roche (1997); es decir, se trata de una relación funcional entre el hacer y la especificación de los criterios del hacer.
Todos los actos de conocimiento son básicamente actos de diferenciación que se realizan como prácticas sociales. Esto es, de delimitaciones o categorización de los haceres. Como lo expresa Ribes (2010), conocer implica actos, pero no se trata de actos como razonar, juzgar, pensar u otros de este estilo y uso en las teorías mentalistas; sino que se trata de actos que pueden situarse en las categorías de espacio y tiempo y que además toman el carácter de conocimiento cuando se establecen las categorías analíticas que le darán a los distintos actos sus diferentes significados o criterios para el hacer; es decir, el conocimiento se corresponde con los criterios o categorías analíticas que significan u orientan las distintas acciones humanas.

La idea de conocimiento, tanto en Aristóteles (trad. en 1980) como en Platón (trad. en 1993), se refería a un juicio justificado y se consideraba que para que fuera significativo como juicio, debía tener la forma de acción justificada. En aras de la comprensión del presente trabajo, puede entenderse la acción justificada como una autorización para el modo de la acción; específicamente, para actuar de cierta forma y no de otra. Esta "autorización" resulta tanto de las consecuencias resultantes de las leyes naturales como de las prácticas sociales o de los efectos que resulten de la acción que se está justificando.

Hablar de juicios solo tiene sentido como lenguaje, es decir, como un acto lingüístico. Y esto es diferente de la consideración de los actos que hacen las teorías cognoscitivas que hablan de actos como si se tratara de acciones de agentes internos (Palmer \& Donahou, 1992) a los que se les atribuyen cualidades y poderes especiales que se ponen en acción cuando se necesitan; como es el caso de actos como razonar (razonar sobre algo), recordar (recordar algo), juzgar (juzgar algo), donde la mente es la que razona, la que recuerda y la que juzga; y es entonces en un espacio mental en donde suceden dichas operaciones.

La especificación de saber como un acto de conocimiento se realiza como una práctica social mediada lingüísticamente. Para Kantor y Smith (1975), la conducta lingüística es un acto comunicativo - un modo de influir en otro- que implica que quien realiza el acto interactúa tanto con un objeto convencional - el referente- como con un objeto que opera como referido; es decir que se trata de una doble interacción, una condición biestimulacional.

Así, al decir que la conducta lingüística es un acto, se abrió la puerta a una lógica distinta al mecanicismo como lógica propuesta en el renacimiento para explicar el mundo de los objetos no vivos; a cambio, los actos lingüísticos toman sentido en una lógica de lo convencional distinta de la lógica causal. En términos de Wittgenstein (1953/1988), se trata, más bien, de "juegos de lenguaje"; esta concepción 
también elimina la idea de que el lenguaje es un medio para reflejar o nominar la realidad, pues los actos verbales son actividades de distinción que se realizan mediante criterios categoriales y de respuesta diferencial. De este modo, son interacciones, no cosas ni etiquetas para transmitir un mensaje que usan quienes hablan o actúan lingüísticamente; y se trata más bien de la coordinación de acciones dadas entre el referidor y el referido que están mediadas por las acciones que la realidad posibilita —el referente-. Así, "el nivel de competencia del lenguaje que posee un individuo determina su actuación lingüística" (Castro, Mathiesen, Mora, Merino \& Navarro, 2011, p.40).

En una interacción lingüística, como se dijo antes, uno de los objetos, o el objeto estimular con los que se interactúa, corresponde a la persona con quien se habla, a quien se le escribe o se le hacen señas; mientras que el segundo objeto estimular corresponde a aquella parte de la realidad que media la interacción. Sin embargo, hay que aclarar que los objetos estimulares son significativos por las funciones que establecen respecto de algo; por ejemplo, ver corresponde a una función que se establece entre la reactividad biológica y las condiciones estimulares - longitud de onda, para el caso- - y, en esta misma lógica, por ejemplo, si alguien dice "páseme la sal", no está expresando una idea resultado de una experiencia ahora verbalizada, sino que lo que este hablante está haciendo es disponer un evento estimular que tiene una función de respuesta elaborada convencionalmente: "pasar la sal". Y lo mismo ocurre con el oyente, pues se es oyente en la medida que se responde de manera pertinente, esto es, "pasando la sal".

Las interacciones lingüísticas, como funciones convencionales que se institucionalizan en las prácticas sociales configuran los distintos modos de interacción y constituyen lo que Wittgenstein (1953) llama "juegos de lenguaje", al interior de los cuales las acciones adquieren sentido como parte de las funciones de los elementos que componen los distintos juegos de lenguaje.

Para Wittgenstein (1953), un juego de lenguaje es un espacio funcional organizado mediante categorías analíticas - funciones conductuales organizadas de acuerdo a los modos que definen el juego-. Así, los biólogos, por ejemplo, hacen uso de categorías analíticas como "árbol", mediante las cuales delimitan un conjunto de objetos o datos empíricos para, dependiendo de las distintas formas de interacción que los objetos posibiliten, elaborar categorías de mayor o menor cobertura con las cuales dar cuenta de los objetos a explicar.

La ciencia es uno de esos espacios funcionales constituido por categorías que están aún por delimitar de manera precisa. Y, en este sentido, Ribes (2003) propuso para la psicología cuatro categorías: la categoría taxonómica, la operacional, la de medición, y la representacional.

Como se ha dicho antes, una categoría analítica es el resultado de una abstracción en sentido skinneriano. Skinner (1957/1981) se refiere a la abstracción así: "la abstracción es un proceso peculiarmente verbal, debido a que un ambiente no verbal no puede proporcionar las contingencias restringidas que lo necesitan" (p.123). Con el desarrollo de nuevas conceptualizaciones y procedimientos, como los relacionados con la equivalencia de estímulos, se ha hecho posible que las contingencias convencionales se puedan especificar y restringir procedimentalmente, tal como se propone en el presente trabajo.

En esta investigación se proponen las relaciones de equivalencia como un criterio (norma de inclusión) para integrar las distintas funciones de las actividades investigativas en las categorías analíticas - como las planteadas por Ribes (2003) - y para estructurar el campo de conocimiento o disciplina.

Investigadores de la conducta, como Sidman (1986), han propuesto una explicación coherente de expresiones como "los significados, referente y conducta gobernada por reglas" con base en el establecimiento de relaciones, principalmente de las relaciones de equivalencia, que incluyen básicamente lo que la gente dice y las reacciones o respuestas a lo que se dice.

Estas relaciones de equivalencia tienen un amplio uso en las matemáticas y se refieren a que una relación es el producto cartesiano de A x B, donde un elemento " $\mathrm{x}$ " pertenece al conjunto $A, y$ un elemento " $y$ " pertenece al conjunto B. Con base en esto, se establecen las relaciones de equivalencia, que tienen las siguientes propiedades: a) reflexividad, que se refiera a que " $\mathrm{x}$ " se relaciona consigo mismo; b) simetría, que se refiere a que si " $\mathrm{x}$ " se relaciona con " $y$ ", entonces " $y$ " se relaciona con " $\mathrm{x}$ "; $y$ c) transitividad, que se refiere a que si se tiene un producto cartesiano $\mathrm{Ax}$ $\mathrm{B}, \mathrm{B} \times \mathrm{C}$, entonces los elementos " $\mathrm{z}$ " de $\mathrm{C}$, se relacionan con " $x$ ", como también se relaciona con "y".

Sidman (1971) usó esta misma lógica para estudiar algunos fenómenos psicológicos, principalmente los relacionados con el comportamiento verbal. En analogía con las matemáticas, las clases $\mathrm{A}, \mathrm{B}$ y $\mathrm{C}$, que en matemáticas se refieren a un conjunto de elementos, en psicología se constituyen a través del control condicional mediante el cual se constituyen las categorías A, B y C, cuyos elementos, también por medio del control condicional, pueden colocarse en relaciones con otras categorías para formar las relaciones de equivalencia (por ejemplo, la categoría A puede estar constituida por los elementos "x1", "x2" y 
"x3", la categoría B por los elementos "y1", "y2” y “y3", y la categoría C por los elementos " $z 1$ ", " $z 2$ " y " $z 3$ ").

Los procedimientos para constituir las categorías son los de tareas de igualación a la muestra, pues en este se presenta en el centro de un tablero un estímulo muestra (EM), y en la parte inferior del tablero se presentan, por lo general, tres estímulos comparadores (EC) con alguno de los cuales se establece algún tipo de relación convencional o criterio de inclusión que corresponde a una primera fase de entrenamiento. En una segunda fase, se ponen en relación (con el mismo procedimiento) los elementos de la categoría A con los elementos de la categoría $\mathrm{B}$ o $\mathrm{C}$, según el caso. Las investigaciones realizadas con estos procedimientos son ya bastante extensas (Dugdale \& Lowe, 1990; Fiorentine et al., 2013; Hayes, Tilley \& Hayes, 1988; Saunders, Wachter \& Spradlin, 1988).

El concepto de relaciones de equivalencia como concepto matemático se utilizó principalmente con propósitos descriptivos, más que para dar una explicación del origen de dichas relaciones (Sidman, 1994), y, si bien no se hace explicito, se puede deducir que la función explicativa también se origina en las prácticas sociales. Sidman (1994) también considera que las relaciones de equivalencia tienen su origen como un efecto de las contingencias de refuerzo, y que estas relaciones, particulares o agrupadas en clases, resultan del control contextual, y por lo mismo, de historias de refuerzo especificas.

De otra parte, Hayes y Hayes (1989), consideran que las relaciones de equivalencia son solo un ejemplar más en una conducta de relacionar - así como igualar, diferenciar $u$ oponer-, que pueden tratarse como cualquier otra operante, que están también bajo el control contextual, y que pueden ser tratadas como una abstracción en el sentido propuesto por Skinner; es decir, que la abstracción surge a través de las contingencias sociales. Y en este sentido, para explicar funciones complejas de los humanos, como el conocer y el pensar entre otros, Hayes, Barnes-Holmes y Roche (2001) propusieron la Teoría de los Marcos Relacionales (TMR).

Un mirada interconductista de las relaciones de equivalencia considera que estas se originan cuando un evento puede fungir como función de estímulo y, a su vez, establecer en el organismo una función de respuesta; así, la palabra "rojo" puede cumplir una función de estímulo, en cuyo caso, un oyente, al oír la palabra, podría como respuesta indicar o señalar un color específico; pero, asimismo, este mismo oyente podría, en otra ocasión, decir "rojo" para indicar o señalar a un oyente la presencia de dicho color. La coordinación de la función de estímulo y la función de respuesta es a lo que se identifica como propiedad reflexiva en las relaciones de equivalencia; relación que se constituye en la interacción fundamental para el establecimiento de las relaciones de equivalencia - es decir, la propiedad reflexiva se corresponde con lo que se conoce como "nombre", que en el ejemplo sería "rojo"-; para un análisis más amplio, véase Barnes y Roche (1997).

Por otra parte, la propiedad combinatoria, característica de las relaciones de equivalencia, se origina cuando se combinan o intercambian convencionalmente distintas funciones de estímulo con distintas funciones de respuesta (por ejemplo, ante la palabra "rojo", que funge como estímulo, se pueden dar distintas repuestas: se puede hacer un dibujo, indicar o escribir), a lo que Skinner se refiere como un "tacto extendido". Adicionalmente, Hayes et al. (2001) extendieron la propiedad combinatoria no solo a los elementos de las relaciones, sino a las relaciones mismas.

Así, entendido el conocimiento como un conjunto de funciones, o como una red de relaciones de categorías analíticas (entendidas como A, B y C) que delimitan convencionalmente un dominio de conocimiento, tal como es el caso de una disciplina, dichas funciones resultan de las relaciones dadas entre las funciones del estímulo y las funciones de la respuesta para así constituir la categoría $\mathrm{A}$ - o categoría descriptiva - , referida como las "propiedades de los objetos" o funciones de estímulo; y la categoría B — o función de respuesta - de "respuestas diferenciales" mediadas convencionalmente y que corresponderían a la categoría de "sujeto".

En una interpretación nominalista y dualista, a la categoría A se le nominaría como "objeto" y a la categoría B como "sujeto". A la luz de los desarrollos en la investigación en la conducta verbal, B puede entenderse como categoría convencional. De este modo, las categorías analíticas se constituyen en la taxonomía propia de las distintas disciplinas, las cuales permiten organizar las distintas interacciones del organismo con el ambiente, distinguiendo a cada una de ellas con base en principios de reflexividad psicológica. Tal vez este sea el giro copernicano al que Kant (1781/2016) se refirió en su crítica de la razón pura.

Las funciones del estímulo y las funciones de la respuesta constituyen una unidad o función conductual con base en las cuales, cuando se integran a una red de relaciones convencionales, se desarrolla lo que en la vida cotidiana se entiende como conocimiento. Aquí, el conocimiento se concibe como un juego de lenguaje, y el conocimiento especializado se puede entender como un juego disciplinar delimitado por criterios o categorías analíticas que una comunidad científica ha venido construyendo a partir de sus interacciones con la realidad y con los miembros de la comunidad especializada; lo que se hace posible mediante la institucionalización o convencionalización de las funciones conductuales en la medida en que se constituyen modos formalizados de interacción con el otro. 
En este sentido, para dar un ejemplo, cuando una persona en un episodio lingüístico habla del "perro", no está usando una etiqueta para nombrar un objeto, sino que está respondiendo en un episodio interactivo con morfologías convencionales establecidas para el objeto estimular perro- $\mathrm{y}$ de las cuales se actualizarán las funciones de respuesta pertinentes a la situación - las condiciones del episodio verbal-. Así, el conjunto de funciones conductuales que se despliegue situacionalmente, o que se actualice en el tiempo y en un espacio específico, constituye el grado de conocimiento referido a un dominio.

La filosofía y la epistemología, sean de origen empirista o racionalista, han supuesto que el conocimiento se origina en la experiencia; y es a partir de la auto-observación descrita cuando se verbaliza tal experiencia interior. La diferencia entre el racionalismo y el empirismo surge básicamente en cómo se describe la realidad; pues se traduce de una lógica a otra. Es como si dos personas que hablen, una inglés y otra español, se quisieran comunicar ¿cómo lo pueden hacer? No se trata de decir “"'book' se dice 'libro' en español", sino que se trata de que los objetos estimulares "book" y "libro" seleccionen de manera intercambiable un conjunto de interacciones que un individuo $u$ otro realizan cotidianamente con el objeto.

Un explicación del conocimiento como fenómeno psicológico hace referencia a la manera como se establecen, organizan y actualizan las funciones conductuales. De este modo, la psicología ha desarrollado procedimientos que permiten dar cuenta de estos tres aspectos; esto es, mediante las tareas de igualación a la muestra. Dichas tareas también han permitido develar la "trama" de lo social, en tanto que las categorías y los conceptos son modos de coordinación que delimitan los tipos de interacción con los otros, con las cosas y con sí mismos. Tan poderosa es la presencia de las categorías y conceptos como estructuras funcionales del mundo social, que regulan funcionalmente toda práctica teórica y, por consiguiente, todo conocimiento científico o no (Carpio, Pacheco, Flores \& Canales, 2002; Padilla, 2003).

\section{COMENTARIO FINAL}

Una teoría científica tiene como función principal delimitar el campo de fenómenos que el investigador se propone analizar, así como los modos en que se hará el análisis, es decir, con qué conceptos, qué instrumentos y cómo el investigador representará sus análisis. Todo esto es posible únicamente mediante los medios convencionales que el investigador seleccione para el análisis de lo que se propone estudiar, y que una comunidad científica ha avalado como idóneos.
Por lo general las primeras explicaciones que se dan de un suceso son explicaciones metafóricas y reduccionistas respecto de lo establecido, de hecho, la ciencia moderna hace extenso uso de metáforas; tal es el caso de Newton, quien, por ejemplo, se refiere al funcionamiento del universo como "un reloj", e, incluso, en el caso de la ley de la gravedad, que es análoga con a la "atracción dada entre las personas" (Newton, 1687/1974, citado por Cohen, 1980). Igual pude decirse de Darwin, quien se refiere a la selección natural como una analogía de las distintas operaciones de selección que el hombre realiza, entendida como la selección artificial (Young, 1970).

La ciencia siempre ha hecho uso de la metáfora como una primera forma de comprensión. El mismo Newton percibió esta relación y por eso hizo el comentario de que tal vez sea necesario en algún punto abandonar la metáfora y hacer uso del lenguaje literal, que para este científico era el lenguaje de las matemáticas (Weinberg, 2015).

En lo que a este estudio respecta, Ruiz y Luciano (2012), por ejemplo, usaron el paradigma de las relaciones de equivalencia para el estudio experimental del razonamiento analógico. Mientras que Ribes (2003) propuso una taxonomía bajo la cual agrupar las distintas actividades que se despliegan en un análisis científico, que serían: medición, representación y actividades de análisis sistemático o procedimentales. Cada una de las cuales posee una lógica propia, pero en conjunto todas constituyen una práctica científica.

Por otra parte, los trabajos realizados con discriminaciones condicionales que usan tareas de igualación a la muestra han permitido entender lo analítico desde una perspectiva distinta a la planteada por el racionalismo. Específicamente, el racionalismo se refiere a lo analítico como el producto de una entidad llamada "mente" cuyo modo de conocimiento se representa en lenguaje; mientras que los trabajos en discriminaciones condicionales han permitido plantear lo analítico como los modos de relación dados entre las interacciones situadas espacial y temporalmente; interacciones mediadas convencionalmente y entendidas como el tipo de interacción que trasciende el aquí y el ahora y se actualizan en el allá y en el entonces; que es lo que hace del conocimiento un valor que como práctica social trasciende el tiempo presente (Barnes \& Roche, 1997).

Toda la diversidad y especialización de la cultura es el resultado de interacciones que se pueden ubicar espaciotemporalmente; $\mathrm{y}$, además, su continua categorización y re-categorización son producto del desarrollo de las prácticas sociales que en su naturaleza fundante no son nada distinto que prácticas lingüísticas. Los distintos sucesos de la realidad solo son comprensibles si pueden integrarse a una práctica social. Y son diversos los conceptos a través de los cuales se ha hablado de esto, por ejemplo, el "paradigma" 
de Kuhn (1971), los "juegos de lenguaje" de Wittgenstein (1953), o la "raíz metáfora" de Pepper (1942).

\section{REFERENCIAS}

Aristóteles (trad. en 1980). Acerca del alma. Madrid: Gredos.

Barnes, D., \& Roche, B. (1997). A behavior-Analytic approach to behavioral reflexivity. The Psychological Record, 47, 543-572.

Cambell, D. T. (1974). Evolutionary Epistemology. En. P. A. Shilpp, Lasalle \& IL (Eds.) The philosophy of Karl R. Popper (pp.412-463). Open Court.

Carpio, C., Pacheco, V., Flores, C., \& Canales, C. (2002). Aprendizaje de la práctica científica en Psicología. Perspectivas de la psicología experimental, 2. 47-71.

Castro, G. G., Mathiesen de Gregory, M. E., Mora, O., Merino, J. M., \& Navarro, G. (2011). Habilidades lingüísticas y rendimiento académico en escolares talentosos. Revista CES Psicología, 5, 40-55. Recuperado de http://www.redalyc. org/articulo.oa? id=423539471004.

Cohen, I. B. (1980). The Newtonian revolution. Cambridge: Cambridge University Press.

Changeaux, J. P. (1985). Neuronal Man. New York: Pantheon.

Darwin, Ch. (1859/1983). El origen de las especies. Madrid: SARPE.

Dugdale, N., \& Lowe, C. F. (1990). Naming and stimulus equivalence. En D. E. Blackman \& H. Lejeune (Eds.). Behaviour Analysis in Theory and Practice (pp. 115-138). Hove: Lawrence Erlbaum Ass.

Edelman, G. M. (1987). Neural Darwinism: The Theory of Neuronal Group Selection. New York: Basic Books.

Fiorentini, L., Vernis, S., Arismendi, M., Primero, G., Argibay, J. C., Sánchez, F., Tabullo, A., Segura, E., \& Yorio, A. A. (2013). Relaciones de equivalencia de estímulos y relaciones de equivalencia equivalencia: Efectos de la estructura de entrenamiento. International Journal of Psychology and Psychological Therapy, 13, 233-242.

Hayes S. C., Barnes-Holmes, D., \& Roche, B. (2001). Relational frame theory: A post-Skinnerian account of human language and cognition. New York: Kluwer Academic.

Hayes, L. J., Tilley, K. J., \& Hayes, S. C. (1988). Extending equivalence class membership to gustatory stimuli. The Psychological Record, 38, 473-482.

Hayes, S. C., \& Hayes, L. J. (1989). The verbal action of the listener as a basis for ruler-governance. En S. C. Hayes (Ed.), Ruler-governed behavior: Cognition, contingencies, and instructional control (pp.153-190). New York: Plenum.

Kant, I. (1781/2016). Crítica de la razón pura. Madrid: Tecnos.

Kantor. J. R., \& Smith, N. W. (1975). The Science of Psychology: An Interbehavioral Survey. Chicago: Principia Press.
Kuhn, T. S. (1971). La estructura de las revoluciones cientificas. México: Fondo de la cultura económica.

Lorenz, K. (1977). Behind the mirror. London: Methuen.

Padilla, M. A. (2003). Un análisis experimental de la dominancia de categorías teóricas en la práctica científica (Tesis doctoral). Universidad de Guadalajara, México.

Padilla, M. A. (2014). Análisis del tipo de interacciones que ocurren entre investigadores durante la presentación de trabajos académicos. Acta Colombiana de Psicología, 17, 3544. doi: 10.14718/ ACP.2014.17.1.4.

Palmer, D. C., \& Donahoe, J. W. (1992). Essentialism and selectionism in cognitive science and behavior analysis. American Psychologist, 47, 1344-1358.

Pepper, S. C. (1942). World hypotheses. Berkeley and Los Angeles: University of California Press.

Piaget, J. (1977). Psychology and Epistemology: Towards a Theory of Knowledge. New Zealand: Penguin Books.

Piaget, J., \& Garcia, R. (1982). Psicogénesis e historia de la ciencia. Madrid: Siglo XXI.

Peirce, Ch. S. (1878/1935). Scientific Metaphysics. En Charles Hartshorne \& Paul Weiss (Eds.), Collected Papers, vol. 6. Cambridge, MA: Harvard University Press.

Pinker, S. (1997). How the mind work. New York: W.W. Norton \& Company.

Platón (trad. en 1993). Diálogos. Bogotá: Panamericana Editorial Limitada.

Ribes, E. (2003). Concepts and theories: Relation to scientific categories. En C. A. Lattal \& P. N. Chase (Eds.), Behavior Theory and Phylosophy (pp. 147-164). New York: Kluwer Academic.

Ribes, E. (2010). Teoría de la conducta 2: Avances y extensiones. México: Trillas.

Ruiz, F. J., \& Luciano, C. (2012). Relacionar relaciones como modelo analítico-funcional de la analogía y la metáfora. Acta Comportamentalia: Revista Latina de Análisis de Comportamiento, 20, 5-31.

Ryle, G. (1949). The concept of mind. Nueva York: Barnes \& Noble.

Sarmiento, J. C. (2011). Conocer: Una Visión Epistémica. Acta Colombiana de Psicología, 14, 81-97.

Saunders, R. R., Wachter, J., \& Spradlin, J. E. (1988). Establishing auditory stimulus control over an eight-member stimulus class via conditional discrimination procedures. Journal of the Experimental Analysis of Behavior, 49, 95-115.

Sidman, M. (1994). Equivalence relations and behavior: A research story. Boston: Authors Cooperative, Inc.

Sidman, M. (1971). Reading and auditory -visual equivalences. Journal of Speech and Hearing Research, 14, 5-13.

Sidman, M. (1986). Functional analysis of emergent verbal class. En T. Thompson \& M. D. Zeiler (Eds.), Analysis and integration of behavior units (pp. 213-245). Hillsdale, N. J. Lawrence Erlbaum associates. 
Skinner, B. F. (1981). Selection by Consequences. Science, 213, 501-504.

Skinner, B. F. (1957/1981). Conducta Verbal. México: Trillas. Tomás, St. (2009). Compendium of Theology. (Traduccion de Regan, R. J). New York: Oxford University Press.

Wittgenstein, L. (1953/1988). Investigaciones filosóficas. México: UNAM.
Weinberg, S. (2015). Explicar el mundo. Bogotá: Penguin Random Hause.

Young, R. M. (1970). Mind, brain and adaptation in the nineteenth century: Cerebral localization and its biological contex from Gall to ferrier. Oxford: Clarendon Press. 